\title{
DUS Characterization of Tamil Nadu Traditional Rice (Oryza Sativa L.) Varieties
}

\author{
J. Johnny Subakar Ivin, Y. Anbuselvam
}

10.18805/IJARe.A-5907

\begin{abstract}
Background: Twenty-seven landraces of rice (Oryza sativa L.), collected from different parts of Tamil Nadu, were characterised according to the DUS guidelines of the Plant Varieties Protection and Farmers' Rights Authority, GOI.

Methods: Rice accessions were planted with three replications using the randomized block design (RBD) in 2019 at the Plant Breeding Farm, Department of Genetics and plant Breeding, Faculty of Agriculture, Annamalai University.

Result: Observations were recorded on 49 different agro-morphological traits, out of 49 descriptors analysed, six were monomorphic, seventeen were dimorphic, thirteen were trimorphic, seven were tetramorphic and spikelet: density pubescence of lemma, 1000 grain weight of fully developed grains and decorticated grain colour showed five expressive states and lemma and palea colour reported six expressive states. Contemporary research work will be advantageous for breeders to choose the right parent for crop improvement and the researchers for genetic diversity among landraces.
\end{abstract}

Key words: Agronomic traits, DUS, Landraces, Morphological characterization.

\section{INTRODUCTION}

In the world, rice is the second most important cereal crop after the corn. Rice is grown in over one hundred countries and produces more than 700 million tonnages annually, with total harvested area of approximately 158 million hectares (470 million tons of milled rice). Asia produces almost 640 million tons of rice, which accounts for $90 \%$ of the world demand. Rice is the main staple food crop and the most competitive in India (Barua and Saikia 2018). Production reaches to 116.42 million tonnes in 2018-2019 and having an estimate of 120.48 million tonnes in 2019-2020 (Anonymous 2019). India has a rich and wide range of genetic wealth of rice (Anju and singhl 2003). Approximately, 425,500 rice accessions stored in various gene banks on worldwide are possible genetic sources for improving guided crops Chakrabarty et al. (2012) Different studies suggest that India has over 70,000 germplasm accessions and also needs the selection and protection of a large number of wild species (Siddiq 1992). Indigenous rice varieties also have an impact for various conditions such as skin disorders, blood pressure, fever, paralyzes, rheumatism, leukorrhea and for increasing tone of lactation in the Indian states of Karnataka, Madhya Pradesh, Kerala, Tamil Nadu, Uttar Pradesh, Western Ghats and the Himachal Pradesh Ahuja et al. (2008). In addition to providing sources for clearly inherited traits such as resistance or the tolerance to biotic and abiotic stresses, traditional rice varieties also provide genes for complex properties for further improvements in grain quality and yield. Singh et al. (2010) Valuable rice germplasm in this region has rapidly disappeared over the last few years due to the change in the variety spectrum and the usage of paddy fields for non-agricultural purposes Latha et al. (2013). Hence, there is an urgent need for
Department of Genetics and Plant Breeding, Faculty of Agriculture, Annamalai University, Annamalai Nagar-608 002, Tamil Nadu, India.

Corresponding Author: Y. Anbuselvam, Department of Genetics and Plant Breeding, Faculty of Agriculture, Annamalai University, Annamalai Nagar-608 002, Tamil Nadu, India.

Email: yanbuselvam@gmail.com

How to cite this article: Ivin, J.J.S., Anbuselvam, Y. (2021). DUS Characterization of Tamil Nadu Traditional Rice (Oryza Sativa L.) Varieties. Indian Journal of Agricultural Research. DOI: 10.18805/ IJARe.A-5907.

Submitted: 06-09-2021 Accepted: 02-11-2021 Online: 20-12-2021

characterization and conservation of these traditional landraces. In this context, an attempt was made to characterize a set of twenty-seven rice landraces for different morphological and agronomic traits and identify the variability available in the collection.

Government of India has introduced Protection of Plant Varieties and Farmers' Rights (PPV \& FR) Act in 2001. DUSbased morphological characterization (Distinctness, Uniformity and Stability) is the criterion for variety registration under the PPVandFR Act. Hence the programme is aiming for DUS characterization of twenty-seven landraces of Tamil Nadu.

\section{MATERIALS AND METHODS Study site}

Twenty-seven traditional varieties of rice were grown in a Randomized block design with three replications in 2019 at the Department of Genetics and plant Breeding farm situated near Faculty of Agriculture, Annamalai, University. The 
experimental site has the co-ordinates of $11^{\circ} 24^{\prime} \mathrm{N}$ latitude and $79^{\circ} 41^{\prime} \mathrm{E}$ longitude at an altitude of $5.1 \mathrm{~m}$ above the main sea level.

\section{Experiment materials}

The present research experimental material consists of 27 local rice cultivars (Oryza sativa L.) of Tamil Nadu. Relevant common name was gathered from local farmers. (Table 1).

\section{Field plot}

Accessions were grown in a randomized block design with the three replications. Each entry was sown in three rows of 20 plants, at a spacing of $15 \mathrm{~cm}$ between plants and $20 \mathrm{~cm}$ between rows and $1 \mathrm{mt}$ isolation separation was maintained between two entries in each block. Crop was raised by following recommended package of practices. (Manjunatha et al. (2018).

\section{Observations}

The observations were taken for forty-nine morphological and agronomic characters. In these observations, Morphological characterization of twenty-seven landraces of rice was carried out using twelve DUS characters. Visual observations were recorded on remaining thirty-seven characters for single plant basis on ten randomly selected plants in each genotype at appropriate growth stages as reported by Umarani et al. (2017).

\section{RESULTS AND DISCUSSION}

Fourty-nine traits were observed in twenty-seven germplasm to establish distinctiveness among germplasm and these are presented below.

\section{Morphological characterization}

Qualitative characters are important for the definition of plants and are mainly influenced by consumer preference, socio economic scenario and natural selection (Ismaeel et al. 2016). Frequency distribution for 39 qualitative traits is depicted in (Table 2). Most of the morphological characters display variability in different accessions, with the exception of Basal sheath colour, Leaf collar, Leaf collar anthocyanin colouration and Leaf Ligule. A majority of accessions possess green (96.30\%) coleoptile colour, purple colour coleoptiles found in (karupu kavuni) $3.70 \%$ of plant as a unique genotype because more than $95 \%$ of the purple coleoptile colour plant is resistant to insect pest i.e. brown plant hopper (Bph) (Bennet 1994) while in wheat purple colour of coleoptile is reportedly related with resistance to bunt. (Shoeva and Elena, 2015).

The observations for leaf traits were recorded as leaf anthocyanin colouration ( $88 \%$ present), leaf distribution of anthocyanin colouration ( $81 \%$ Uniform), leaf sheath anthocyanin coloration (88\% present), leaf auricles $(85 \%$ Present), leaf anthocyanin coloration of auricles (85\% colourless), leaf colour of ligule ( $96 \%$ white), leaf intensity of green colour ( $40 \%$ Medium, 33\% light, $26 \%$ dark), leaf sheath intensity of anthocyanin coloration (44\% medium,
$30 \%$ weak, $26 \%$ strong), leaf pubescence of blade surface (52\% medium, 33\% strong, 14\% weak,) leaf shape of ligule (52\% acute, $30 \%$ split, $18 \%$ truncate), Similar type of work was also reported (Bisne and Sarawgi, 2008; Ahmed et al. 2016) and some of the accessions possessed various distributions were found in (Shrivastava et al., 2015). Culm attitude is an indicator of the growth habit of a particular species. During the current study valuable variation was observed among the accessions for culm angle. (Rawte and Saxena, 2018). Culm attitude (for floating rice) ( $81 \%$ procurement), culm attitude (40\% erect, $19 \%$ semi erect, $4 \%$ spreading) (Fonseca et al., 2002). Time of heading $50 \%$ of plants with panicles were observed and noticed that $52 \%$ landraces are medium type, 44\% landraces are very late type and $3 \%$ (Arupatham kuruvai) landraces were of early and a short duration variety. Flag leaf: attitude of blade (early observation) (48\% semi erect, $30 \%$ horizontal, $18 \%$ erect, $3 \%$ drooping), Spikelet: density of pubescence of lemma (55\% absent, $18 \%$ medium, $14 \%$ strong, $7 \%$ weak, $3 \%$ very strong), Spikelet: colour of stigma (30\% light green, $7 \%$ yellow and purple) (Nascimento et al. 2011) Stem: Thickness (59\% thick, $29 \%$ thin, $11 \%$ medium), Panicle: length of main axis (19\% medium, $15 \%$ very short, $7 \%$ long and very long), Flag leaf: attitude of blade (late observation) (19\% semi

Table 1: List of genotypes and the place of collection.

\begin{tabular}{ll}
\hline Name of the Genotypes & Place of collection \\
Karupu Kavuni & Thirukadaiyur, Nagapattinam \\
Illapai Poo Samba & Thirukadaiyur, Nagapattinam \\
Kattu Yanam & Vellapallam Nagapattinam \\
Arcode Kichili Samba & Ediyure, Kancheepuram \\
Sorna Masuri & Chembudai, Nagapatinam \\
Seeraga Samba & Coastal area, Nagapatiinam \\
Thooyamalli & Sukan Kollai, Kanchipuram \\
Navara & Vellapallam Nagapattinam \\
Mapillai Samba & Sukkankollai, Kanchipuram \\
Mysore Samba & Sukkankollai, Kanchipuram \\
Karudan Samba & Ananthapuram Tiruvannamalai \\
Milagu Samba & Kanchipuram \\
Kattue Vallai & Vellapallam Nagapattinam \\
Kuzhi Adichan & Denkanikottai krishnagiri \\
Salam Sanna & Mangalam, Kancheepuram \\
Arupatham Kuruvai & Sivagangai \\
Kichili Samba & Ediyure, Kancheepuram \\
Kalan Namak & Sukkankollai, Kanchipuram \\
Poonkar & Ramanathapuram \\
Karunkuruvai & Ananthapuram Tiruvannamalai \\
Kattanure & Vellapallam Nagapattinam \\
Sivapu Chittirai Kar & Anumantankadi, Sivagangai \\
Vallai Chittrai Kar & Sukan Kollai, Kanchipuram \\
Matti Kar & Thirukadaiyur, Nagapattinam \\
Norungan & Coastal area, Nagapatiinam \\
Nootripathu & Coastal area, Nagapatiinam \\
Kuruvai Kalanjium & Thiruppurambiam, Kumbakonam \\
\hline &
\end{tabular}


DUS Characterization of Tamil Nadu Traditional Rice (Oryza Sativa L.) Varieties

Table 2: Frequency distribution of landraces of rice for various DUS characters.

\begin{tabular}{|c|c|c|c|c|}
\hline Characteristics & States & Scale & Number of genotypes & Frequency distribution(\%) \\
\hline \multirow[t]{3}{*}{ Coleoptile: Colour } & Colorless & 1 & 00 & 00 \\
\hline & Green & 2 & 26 & 96.30 \\
\hline & Purple & 3 & 01 & 3.70 \\
\hline \multirow[t]{4}{*}{ Basal leaf: Sheath colour } & Green & 1 & 27 & 100 \\
\hline & Light purple & 2 & 00 & 00 \\
\hline & Purple lines & 3 & 00 & 00 \\
\hline & Uniform purple & 4 & 00 & 00 \\
\hline \multirow{3}{*}{$\begin{array}{l}\text { Leaf: Intensity of } \\
\text { green colour }\end{array}$} & Light & 3 & 09 & 33.33 \\
\hline & Medium & 5 & 11 & 40.74 \\
\hline & Dark & 7 & 07 & 25.92 \\
\hline \multirow{6}{*}{$\begin{array}{l}\text { Leaf: Anthocyanin } \\
\text { colouration } \\
\text { Leaf: Distribution of } \\
\text { anthocyanin colouration }\end{array}$} & Absent & 1 & 03 & 11.11 \\
\hline & Present & 9 & 24 & 88.88 \\
\hline & On tips only & 1 & 02 & 7.40 \\
\hline & On margins only & 2 & 00 & 00 \\
\hline & In blotches only & 3 & 00 & 00 \\
\hline & Uniform & 4 & 22 & 81.48 \\
\hline Leaf sheath: & Absent & 1 & 03 & 11.11 \\
\hline Anthocyanin colouration & Present & 9 & 24 & 88.88 \\
\hline \multirow{5}{*}{$\begin{array}{l}\text { Leaf sheath: Intensity of } \\
\text { anthocyanin colouration }\end{array}$} & Very weak & 1 & 00 & 00 \\
\hline & Weak & 3 & 08 & 29.62 \\
\hline & Medium & 5 & 12 & 44.44 \\
\hline & Strong & 7 & 07 & 25.92 \\
\hline & Very strong & 9 & 00 & 00 \\
\hline \multirow{5}{*}{$\begin{array}{l}\text { Leaf: Pubescence of } \\
\text { blade surface }\end{array}$} & Absent & 1 & 00 & 00 \\
\hline & Weak & 3 & 04 & 14.81 \\
\hline & Medium & 5 & 14 & 51.85 \\
\hline & Strong & 7 & 09 & 33.33 \\
\hline & Very strong & 9 & 00 & 00 \\
\hline \multirow[t]{2}{*}{ Leaf: Auricles } & Absent & 1 & 04 & 14.81 \\
\hline & Present & 9 & 23 & 85.18 \\
\hline Leaf: Anthocyanin & Colorless & 1 & 23 & 85.18 \\
\hline \multirow[t]{2}{*}{ coloration of auricles } & Light purple & 2 & 00 & 00 \\
\hline & Purple & 3 & 01 & 3.70 \\
\hline \multirow[t]{2}{*}{ Leaf: Collar } & Absent & 1 & 00 & 00 \\
\hline & Present & 9 & 27 & 100 \\
\hline Leaf: Anthocyanin & Absent & 1 & 00 & 00 \\
\hline coloration of collar & Present & 9 & 27 & 100 \\
\hline \multirow[t]{2}{*}{ Leaf: Ligule } & Absent & 1 & 00 & 00 \\
\hline & Present & 9 & 27 & 100 \\
\hline \multirow[t]{3}{*}{ Leaf: Shape of ligule } & Truncate & 1 & 05 & 18.51 \\
\hline & Acute & 2 & 14 & 51.85 \\
\hline & Split & 3 & 08 & 29.62 \\
\hline \multirow[t]{3}{*}{ Leaf: Colour of ligule } & White & 1 & 26 & 96.29 \\
\hline & Light purple & 2 & 00 & 00 \\
\hline & Purple & 3 & 01 & 3.70 \\
\hline \multirow[t]{3}{*}{ Leaf: Length of blade } & Short $(<30 \mathrm{~cm})$ & 3 & 05 & 18.51 \\
\hline & Med. $(30-45 \mathrm{~cm})$ & 5 & 13 & 48.14 \\
\hline & Long (>45 cm) & 7 & 09 & 33.33 \\
\hline \multirow[t]{3}{*}{ Leaf: Width of blade } & Narrow $(<1 \mathrm{~cm})$ & 3 & 16 & 59.25 \\
\hline & Medium $(1-2 \mathrm{~cm})$ & 5 & 04 & 14.81 \\
\hline & Broad $(>2 \mathrm{~cm})$ & 7 & 07 & 25.92 \\
\hline
\end{tabular}


DUS Characterization of Tamil Nadu Traditional Rice (Oryza Sativa L.) Varieties

Table 2: Continue...

\begin{tabular}{|c|c|c|c|c|}
\hline \multirow[t]{2}{*}{ Culm: Attitude (for floating rice only) } & Non procumbent & 1 & 05 & 18.51 \\
\hline & Procumbent & 9 & 22 & 81.48 \\
\hline \multirow[t]{4}{*}{ Culm: Attitude } & Erect & 1 & 11 & 40.74 \\
\hline & Semi-erect & 3 & 05 & 18.51 \\
\hline & Open & 5 & 00 & 00 \\
\hline & Spreading & 7 & 01 & 3.70 \\
\hline \multirow{5}{*}{$\begin{array}{l}\text { Time of heading (50\% of plants } \\
\text { withpanicles) (days) }\end{array}$} & Very early $(<71)$ & 1 & 00 & 00 \\
\hline & Early $(71-90)$ & 3 & 01 & 3.70 \\
\hline & Medium (91-110) & 5 & 14 & 51.85 \\
\hline & Late $(111-130)$ & 7 & 00 & 00 \\
\hline & Very late $(>131)$ & 9 & 12 & 44.44 \\
\hline \multirow{4}{*}{$\begin{array}{l}\text { Flag leaf: Attitude of blade } \\
\text { (early observation) }\end{array}$} & Erect & 1 & 05 & 18.51 \\
\hline & Semi-erect & 3 & 13 & 48.14 \\
\hline & Horizontal & 5 & 08 & 29.62 \\
\hline & Drooping & 7 & 01 & 3.70 \\
\hline \multirow{5}{*}{$\begin{array}{l}\text { Spikelet: Density of } \\
\text { pubescence of lemma }\end{array}$} & Absent & 1 & 15 & 55.55 \\
\hline & Weak & 3 & 02 & 7.40 \\
\hline & Medium & 5 & 05 & 18.51 \\
\hline & Strong & 7 & 04 & 14.81 \\
\hline & Very strong & 9 & 01 & 3.70 \\
\hline Lemma: Anthocyanin & Absent/very weak & 1 & 14 & 51.85 \\
\hline \multirow[t]{4}{*}{ colouration of keel } & Weak & 3 & 01 & 3.70 \\
\hline & Medium & 5 & 01 & 3.70 \\
\hline & Strong & 7 & 03 & 11.11 \\
\hline & Very strong & 9 & 00 & 00 \\
\hline \multirow{5}{*}{$\begin{array}{l}\text { Lemma: Anthocyanin colouration } \\
\text { of area belowapex }\end{array}$} & Absent & 1 & 14 & 51.85 \\
\hline & Weak & 3 & 01 & 3.70 \\
\hline & Medium & 5 & 01 & 3.70 \\
\hline & Strong & 7 & 03 & 11.11 \\
\hline & Very strong & 9 & 00 & 00 \\
\hline \multirow{5}{*}{$\begin{array}{l}\text { Lemma: Anthocyanin } \\
\text { colouration of apex }\end{array}$} & Absent & 1 & 14 & 51.85 \\
\hline & Weak & 3 & 01 & 3.70 \\
\hline & Medium & 5 & 01 & 3.70 \\
\hline & Strong & 7 & 03 & 11.11 \\
\hline & Very strong & 9 & 00 & 00 \\
\hline \multirow{5}{*}{$\begin{array}{l}\text { Spikelet: Colour } \\
\text { of stigma }\end{array}$} & White & 1 & 00 & 00 \\
\hline & Light green & 2 & 08 & 29.62 \\
\hline & Yellow & 3 & 02 & 7.40 \\
\hline & Light purple & 4 & 00 & 00 \\
\hline & Purple & 5 & 02 & 7.40 \\
\hline \multirow[t]{3}{*}{ Stem: Thickness } & Thin $(<0.40 \mathrm{~cm})$ & 3 & 08 & 29.62 \\
\hline & Medium $(0.40-0.55 \mathrm{~cm})$ & 5 & 03 & 11.11 \\
\hline & Thick $(>0.55 \mathrm{~cm})$ & 7 & 16 & 59.25 \\
\hline \multirow{5}{*}{$\begin{array}{l}\text { Stem: Length (excluding panicle; } \\
\text { excluding floating rice) }\end{array}$} & Very short $(<91 \mathrm{~cm})$ & 1 & 12 & 44.44 \\
\hline & Short $(91-110 \mathrm{~cm})$ & 3 & 15 & 55.55 \\
\hline & Medium $(111-130 \mathrm{~cm})$ & 5 & 00 & 00 \\
\hline & Long $(131-150 \mathrm{~cm})$ & 7 & 00 & 00 \\
\hline & Very long $(>150 \mathrm{~cm})$ & 9 & 00 & 00 \\
\hline \multirow{5}{*}{$\begin{array}{l}\text { Stem: Anthocyanin } \\
\text { colouration of nodes } \\
\text { Stem: Intensity of anthocyanin } \\
\text { colouration of nodes }\end{array}$} & Absent & 1 & 19 & 70.37 \\
\hline & Present & 9 & 08 & 29.62 \\
\hline & Weak & 3 & 02 & 7.40 \\
\hline & Medium & 5 & 01 & 3.40 \\
\hline & Strong & 7 & 05 & 18.51 \\
\hline
\end{tabular}


Table 2: Continue...

\begin{tabular}{|c|c|c|c|c|}
\hline Stem: Anthocyanin colouration & Absent & 1 & 05 & 18.51 \\
\hline of internodes & Present & 9 & 08 & 29.62 \\
\hline \multirow[t]{5}{*}{ Panicle: Lengthof main axis } & Very short $(<16 \mathrm{~cm})$ & 1 & 04 & 14.81 \\
\hline & Short $(16-20 \mathrm{~cm})$ & 3 & 00 & 00 \\
\hline & Medium $(21-25 \mathrm{~cm})$ & 5 & 05 & 18.51 \\
\hline & Long $(26-30 \mathrm{~cm})$ & 7 & 02 & 7.40 \\
\hline & Very long $(>30 \mathrm{~cm})$ & 9 & 02 & 7.40 \\
\hline \multirow{4}{*}{$\begin{array}{l}\text { Flag leaf: Attitude of blade } \\
\text { (late observation) }\end{array}$} & Erect & 1 & 04 & 14.81 \\
\hline & Semi-erect & 3 & 05 & 18.51 \\
\hline & Horizontal & 5 & 01 & 3.40 \\
\hline & Deflexed & 7 & 01 & 3.40 \\
\hline \multirow[t]{4}{*}{ Panicle: Curvature of main axis } & Straight & 1 & 04 & 14.81 \\
\hline & Semi-straight & 3 & 05 & 18.51 \\
\hline & Deflexed & 5 & 00 & 00 \\
\hline & Dropping & 7 & 02 & 7.40 \\
\hline \multirow[t]{3}{*}{ Panicle: Numberper plant } & Few $(<11)$ & 3 & 06 & 22.22 \\
\hline & Medium (11-20) & 5 & 05 & 18.51 \\
\hline & Many $(>20)$ & 7 & 00 & 00 \\
\hline \multirow[t]{6}{*}{ Spikelet: Colourof tip of lemma } & White & 1 & 00 & 00 \\
\hline & Yellowish & 2 & 02 & 7.40 \\
\hline & Brown & 3 & 04 & 14.81 \\
\hline & Red & 4 & 00 & 00 \\
\hline & Purple & 5 & 01 & 3.70 \\
\hline & Black & 6 & 04 & 14.81 \\
\hline \multirow[t]{9}{*}{ Lemma and Palea: Colour } & Straw & 1 & 01 & 3.40 \\
\hline & $\begin{array}{l}\text { Gold and gold furrows } \\
\text { on strawbackground }\end{array}$ & 2 & 01 & 3.40 \\
\hline & Brown spots on straw & 3 & 00 & 00 \\
\hline & Brown furrows on straw & 4 & 00 & 00 \\
\hline & Brown (tawny) & 5 & 01 & 3.40 \\
\hline & Reddish to light purple & 6 & 01 & 3.40 \\
\hline & Purple spots/furrows on straw & 7 & 03 & 11.11 \\
\hline & Purple & 8 & 00 & 00 \\
\hline & Black & 9 & 03 & 11.11 \\
\hline \multirow[t]{2}{*}{ Panicle: Awns } & Absent & 1 & 01 & 3.40 \\
\hline & Present & 9 & 01 & 3.40 \\
\hline \multirow{2}{*}{$\begin{array}{l}\text { Panicle: Presence of } \\
\text { secondary branching }\end{array}$} & Absent & 1 & 02 & 7.40 \\
\hline & Present & 9 & 08 & 29.62 \\
\hline \multirow[t]{3}{*}{ Panicle: Secondary branching } & Weak & 1 & 06 & 22.22 \\
\hline & Strong & 2 & 02 & 7.40 \\
\hline & Clustered & 3 & 00 & 00 \\
\hline \multirow[t]{6}{*}{ Panicle: Attitude of branches } & Erect & 1 & 00 & 00 \\
\hline & Erect to semi-Erect & 3 & 00 & 00 \\
\hline & Semi-erect & 5 & 02 & 7.40 \\
\hline & Semi-erect to & & 00 & 00 \\
\hline & spreading & 7 & 00 & 00 \\
\hline & Spreading & 9 & 00 & 00 \\
\hline \multirow[t]{3}{*}{ Panicle: Exsertion } & Partly exserted & 3 & 03 & 11.11 \\
\hline & Mostly exserted & 5 & 00 & 00 \\
\hline & Well exserted & 7 & 05 & 18.51 \\
\hline \multirow{3}{*}{$\begin{array}{l}\text { Grain: Weight of } 1000 \text { fully } \\
\text { developed grains }\end{array}$} & Very low $(<15 \mathrm{~g})$ & 1 & 02 & 7.40 \\
\hline & Low (15-20 g) & 3 & 04 & 14.81 \\
\hline & Medium (21-25 g) & 5 & 05 & 18.51 \\
\hline
\end{tabular}


DUS Characterization of Tamil Nadu Traditional Rice (Oryza Sativa L.) Varieties

\begin{tabular}{|c|c|c|c|c|}
\hline & High (26-30) & 7 & 02 & 7.40 \\
\hline & Very high (>30 g) & 9 & 01 & 3.40 \\
\hline \multirow[t]{5}{*}{ Grain:length } & Very short $(<6.0 \mathrm{~mm})$ & 1 & 27 & 100 \\
\hline & Short $(6.1-8.5 \mathrm{~mm})$ & 3 & 00 & 00 \\
\hline & Medium (8.6-10.5 mm) & 5 & 00 & 00 \\
\hline & Long (10.6-12.5 mm) & 7 & 00 & 00 \\
\hline & Very long (>12.5 mm) & 9 & 00 & 00 \\
\hline \multirow[t]{5}{*}{ Grain:width } & Very narrow $(<2.0 \mathrm{~mm})$ & 1 & 25 & 92.59 \\
\hline & Narrow (2.1-2.5 mm) & 3 & 02 & 7.40 \\
\hline & Medium (2.6-3.0 mm) & 5 & 00 & 00 \\
\hline & Broad (3.1-3.5 mm) & 7 & 00 & 00 \\
\hline & Very broad (>3.5 mm) & 9 & 00 & 00 \\
\hline \multirow[t]{7}{*}{ Decorticated grain: Length } & Short & 1 & 14 & 51.85 \\
\hline & Medium & 3 & 12 & 44.44 \\
\hline & Long & 5 & 01 & 3.40 \\
\hline & Long* $^{*}$ & 7 & 00 & 00 \\
\hline & (Long for Basmati type) Extra long & 9 & 00 & 00 \\
\hline & Medium $(2.0-2.5 \mathrm{~mm})$ & 5 & 19 & 70.37 \\
\hline & Broad $(>2.5 \mathrm{~mm})$ & 7 & 00 & 00 \\
\hline \multirow{8}{*}{$\begin{array}{l}\text { Decorticated grain: Shape } \\
\text { (in lateral view) }\end{array}$} & Short slender & 1 & 17 & 62.96 \\
\hline & Short bold & 2 & 00 & 00 \\
\hline & Medium slender & 3 & 01 & 3.70 \\
\hline & Long bold & 4 & 00 & 00 \\
\hline & Long slender & 5 & 06 & 22.22 \\
\hline & Long slender* & 6 & 03 & 11.11 \\
\hline & (For Basmati type) & & & \\
\hline & Extra-long slender & & & \\
\hline \multirow[t]{9}{*}{ Decorticated grain: colour } & White & 1 & 07 & 25.92 \\
\hline & Light brown & 2 & 12 & 44.44 \\
\hline & Variegated brown & 3 & 02 & 7.40 \\
\hline & Dark brown & 4 & 03 & 11.11 \\
\hline & Light red & 5 & 03 & 11.11 \\
\hline & Red & 6 & 00 & 00 \\
\hline & Variegated purple & 7 & 00 & 00 \\
\hline & Purple & 8 & 00 & 00 \\
\hline & Dark purple & 9 & 00 & 00 \\
\hline
\end{tabular}

erect, $15 \%$ erect, $3 \%$ horizontal and deflexed), panicle curvature of main axis (19 semi straight, 15\% straight, $7 \%$ drooping), spikelet colour of tip of lemma (15\% brown and black, $7 \%$ yellowish, $4 \%$ purple, 3\% straw), Lemma and Palea: colour (11\% purple and black, $3.40 \%$ reddish, gold and brown), Panicle: presence of secondary branching (30\% present, 7\% absent) (Ndour et al. 2016), Panicle: secondary branching (22\% weak, $7 \%$ strong), In the traditional growing areas of Asia, rices of various colors-red, black, brown, yellow and green have been known and grown, but for the present day generation, rice connotes pearly white grain. Colored rices have been preferred in the past for their special features such as medicinal value and exclusive taste. Flavoured, black rices were the favourites of the royals of China, while red rices were preferred by people in many parts of India, Sri Lanka and Bhutan. (Rood, 2000)
Decorticated grain: colour (44\% light brown, 30\% white, $11 \%$ dark brown and light red, 7\% variegated brown) (Rao et al., 2013; Tirkey et al., 2013; Mondal et al., 2014; Manjunatha et al., 2016; Kalyan et al., 2017; Umarani et al., 2017) and similar results has been reported earlier and some morphological characters showed similar type of variations in different accessions such as Lemma: anthocyanin colouration of keel, Lemma: anthocyanin colouration of area below apex, Lemma: anthocyanin colouration of apex.

\section{Agronomic characterization}

The quantitative characters also showed wide variation among all varieties (Table 2). Most of the Agronomic characters exhibited variations in various accessions except grain length. A majority of accessions were found to possess Grain: width (92.59\% very narrow) and the balance $7.40 \%$ 


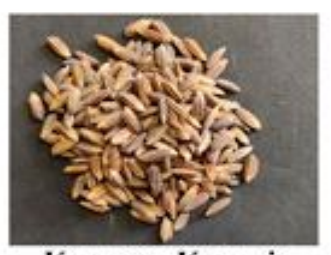

Karuppu Kavuni

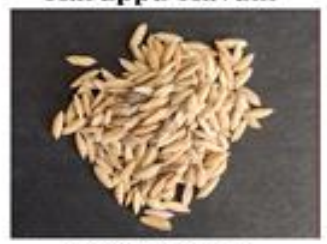

Sorna Masuri

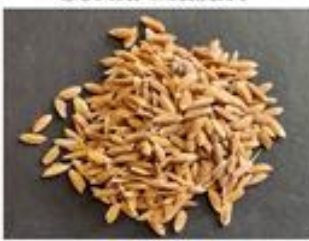

Mapillai Samba

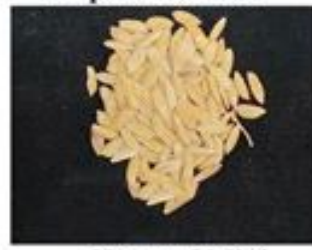

Kattue Vallai

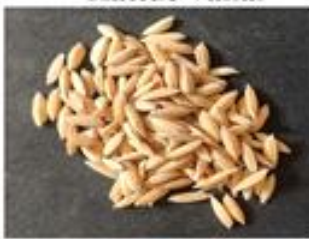

Kichili Samba

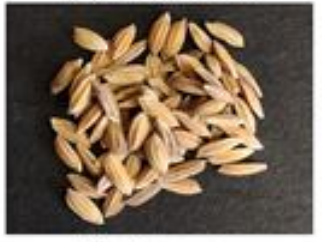

Kattanure

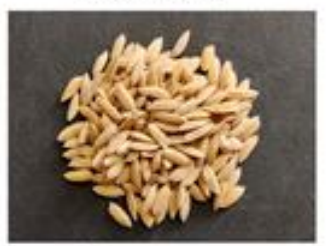

Norungan

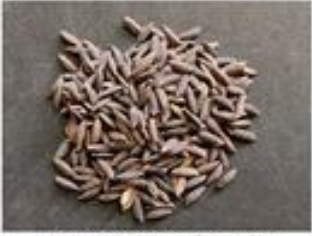

IIlupai Poo Samba

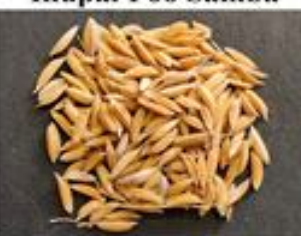

Seeraga Samba

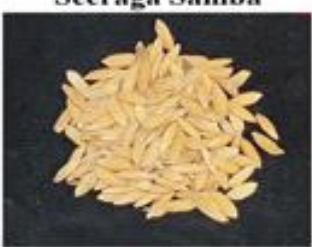

Mysore Malli

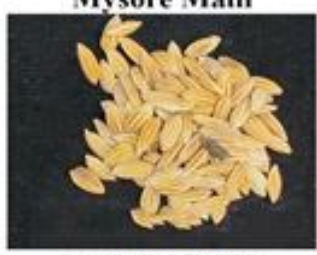

Kuzhiadichan

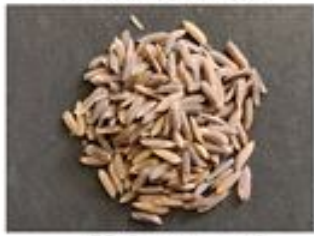

Kalan namak
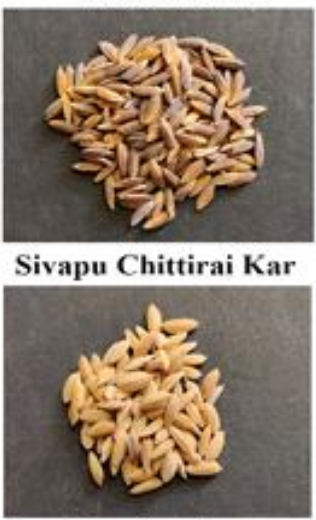

Nootripathu

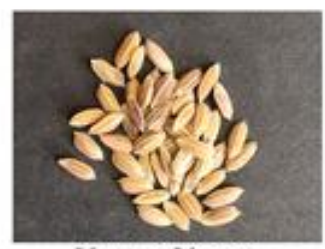

Kattue Yanam

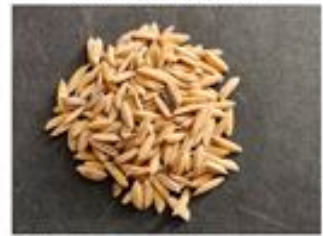

Thooya Malli

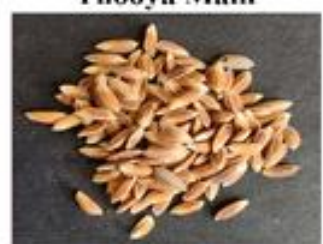

Karudan Samba

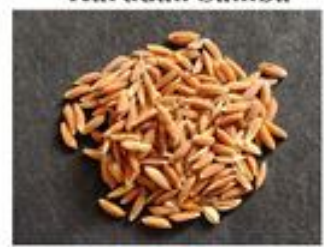

Salem Sanna

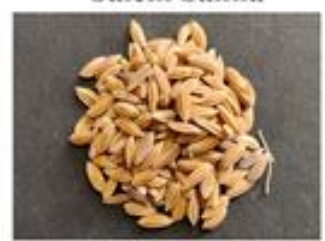

Poonkar

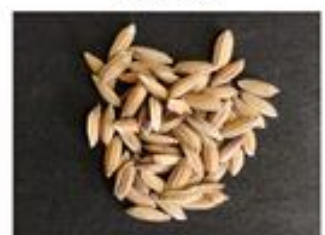

Vellai Chittirai Kar

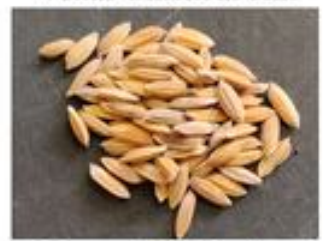

Kuruvai Kalanjium

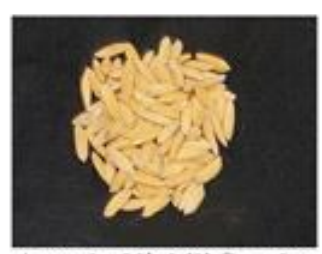

Arcode Kichili Samba

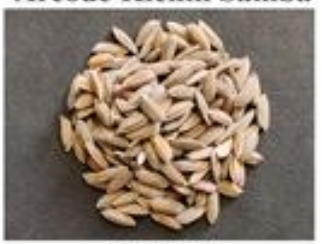

Navara

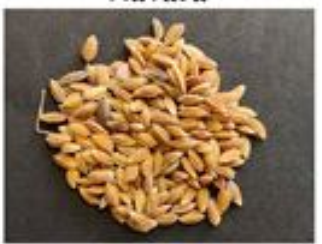

Milagu Samba

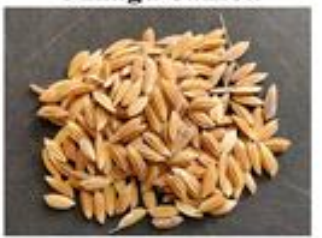

Arupatham Kuruvai

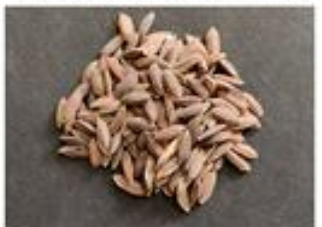

Karun Kuruvai

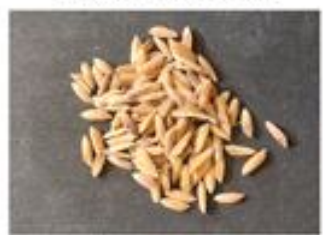

Matti Kar

Fig 1: Evaluation of indigenous rice cultivars from Tamil Nadu.

narrow genotypes possess kattu yanam and karudan samba. Leaf: length of blade (48\% medium, 33\% long, $19 \%$ short), Leaf: width of Blade (59\% narrow, $26 \%$ broad, $15 \%$ medium), Stem: length (excluding panicle; excluding floating rice) (55\% short), Panicle: number per plant (22\% few, $19 \%$ medium), Grain: weight of 1000 fully developed grains consist of $19 \%$ medium, $15 \%$ low, $7 \%$ high, $7 \%$ (Illaapai poo samba (14.59 gram) and Milagu samba (14.84 gram) very low) and 3\% (Mapillai Samba (30.41 gram) very high). By compare with the hybrid varieties, hybrid had higher grain weight than inbred varieties but traditional rice varieties have more over high in inbred and hybrid varieties. (Lu et al., 2020). 
According to the DUS protocol decorticated rice grain length and width have been grouped into three categories short grains, medium grain and long grain, in decorticated grain width $70 \%$ of the accessions possessed medium and others were found various distributions. Decorticated grain: length possessed $52 \%$ short, $44 \%$ medium, $3 \%$ long. Seed shape have been used to differentiate the rice genotypes by various researchers (Bhattacharya and sowbhagya 1980) Decorticated grain: shape $(63 \%$ of the varieties are short slender, $22 \%$ long slender and $11 \%$ possess Long slender (For Basmati type) Extra-long slender variety (sorna masuri, kattue vallai and kuzhiadichan) (Komala et al. 2017).

\section{CONCLUSION}

The identified variability and unique characteristics can be utilized for the development of varieties. Unique genotypes can be registered in PPV and FRA. Qualitative features such as dark green leaf colour, heavy pubescence on the leaf blade or purple surface, margin leaf blade etc. have been identified (Karuppu kavuni) and utilized for developing rice varieties with tolerant to leaf surface, related insects and diseases.

\section{ACKNOWLEDGEMENT}

Authors are thankful to the Department of Genetics and Plant Breeding, Faculty of Agriculture, Annamalai University, Tamil Nadu, India for providing the Experimental farm facilities for the conduct of present study.

\section{Conflict of interests}

The authors declare that there is no competing interest.

\section{REFERENCES}

Ahmed, M.S.U., Bashar, M.K., Wazuddin, M. and Shamsuddin, A.K.M. (2016). Agro-morphological qualitative characterization of Jesso-Balam rice (Oryza sativa L.) accessions in Bangladesh. International Journal of Agronomy and Agricultural Research. 8(1): 50-58.

Ahuja, U., Ahuja, S.C., Thakrar, R. and Singh, R.K. (2008). Rice-a nutraceutical. Asian Agric. Hist. 12: 93-108.

Anju, Singh, M. and Singh, S.P. (2003). Indian rice genetic resources and their contribution to world rice improvement - A review. Agricultural reviews (24): 292-297.

Anonymous (2019). Rice - Statistics and Facts. Statista. https:// www.statista.com/topics rice/.

Barua, D. and Saikia, M. (2018). Agronomic biofortification in rice varieties through zinc fertilization under aerobic condition. Indian Journal of Agricultural Research. 52(1): 89-92.

Bennett, J. (1994). Current status of rice biotechnology in research and application. Plant Biotechnology in Technical Cooperation Programmes.

Bhattacharya, K.R., Sowbhagya, C.M. and Indudhara, S. (1980). Quality of Indian rice. Journal of Food Science and Technology. 17(4): 189-193.

Bisne, R. and Sarawgi, A.K. (2008). Agro-morphological and quality characterization of Badshah bhog group from aromatic rice germplasm of Chhattisgarh, Bangladesh. J. Agril. Res. 33(3): 479-492.

Chakrabarty, S.K., Monika, A.J., Singh, Y., Maity, A., Vashisht, V. and Dadlani, M. (2012). Characterization and evaluation of variability in farmers' varieties of rice from West Bengal. Indian journal of Genetics and Plant Breeding. 72(2): $136-142$.

Fonseca, J.R., Cutrim, V.A. and Rangel, P.H.N. (2002). Morpho Agronomic Descriptors and Phenology of the Lowland Rice Cultivars. Embrapa, Brasília, DF. Brazil (in Portuguese).

Ismaeel, M., Ali Shah, S.M., Nawaz, A., Rehman Suliman, A.U.S. and Khan, A. (2016). Morphological diversity of 83 rice accessions for qualitative and quantitative parameters. International Journal of Bioscience. 9(3): 158-169.

Kalyan, B., Krishna, K.V.R and Rao, S.L.V. (2017). DUS characterization for germplasm of rice. Int J. Curr Microbiol App Sci. 6: 3480-3487.

Komala, N.T., Gurumurthy, R. and Surendra, P. (2017). Morphological characterization of popular rice varieties of Zone VIII and Zone IX of Karnataka State. Bull. Env. Pharmacol. Life Sci. Vol 6 Special issue. 515-519.

Latha, M., Nizar, A.M., Abraham, Z., John, J.K., Nair, R.A., Mani, S. and Dutta, M. (2013). Rice landraces of Kerala state of India: A documentation. Int J. Biodivers Conserv. 5: 250-263.

Lu, J., Wang, D., Liu, K., Chu, G., Huang, L., Tian, X. and Zhang, Y. (2020). Inbred varieties outperformed hybrid rice varieties under dense planting with reducing nitrogen. Scientific Reports. 10(1): 1-13.

M.A. Rood. (2000). Red menace. Rice Journal. 103: 18-20.

Manjunatha, G.A., Elsy, C.R., Rajendran, P., Francies, J.J.R.M. and Krishnan, S. (2018). DUS Characterization of rice (Oryza sativa L.) landraces of wayanad, kerala. Electronic Journal of Plant Breeding. 9(2): 617-630.

Manjunatha, G.A., Vanaja, T., Naik, J., Kumar, A.S.A. and Vasudevan, N.R. (2016). Identification of rice genotypes best suited for the development of organic varieties and identification of current varieties best suited for organic farming. $\mathrm{J}$ Organics. 3(1): 16-24.

Mondal, B., Singh, S.P. and Joshi, D.C. (2014). DUS characterization of rice (Oryza sativa L.) using morphological descriptors and quality parameters. Outlook on Agric. 43: 131-137.

Nascimento, W.F. Silva, E.F. and Veasey, E.A. (2011). Agro morphological characterization of upland rice accessions. Scientific Agriculture (Piracicaba Braz.). 68 (6): 652-60.

Ndour, D., Diouf, D., Bimpong, I.K., Sow, A., Kanfany, G. and Manneh, B. (2016). Agro-Morphological Evaluation of Rice (Oryza sativa L.) for Seasonal Adaptation in the Sahelian, Environment. Agronomy. 6(8).

Rao, L.V.S., Prasad, G.S., Chiranjivi, M., Chaitanya, U. and Surendhar, R. (2013). DUS Characterization for Farmer varieties of rice. IOSR Journal of Agriculture and Veterinary Science (IOSR-JAVS). 5(4): 35-43.

Rawte, S. and Saxena, R.R. (2018). Morphological characterization of selected rice (Oryza sativa L.) from core germplasm 
group of Chhattisgarh using DUS descriptors. Int. J. Curr. Microbiol. App. Sci. 7(10): 350-357.

Shobha Rani, N., Shobha Rao, L.V., Viraktamath, B.C. and Mishra, B. (2006). National Guidelines for the Conduct of Tests for Distinctiveness, Uniformity and Stability. Directorate of Rice Research. 6-13.

Shoeva, O.Y. and Khlestkina, E.K. (2015). The specific features of anthocyanin biosynthesis regulation in wheat. In Advances in wheat genetics: from genome to field Springer. Tokyo. (pp. 147-157).

Shrivastava, A., Koutu, G.K., Mishra, D.K. and Singh, S.K. (2015). Characterization of JNPT lines of rice (Oryza sativa L.). Plant Archives. 15(1): 397-403.
Siddiq, E.A. (1992). Rice in India-potential areas for research and development. Oryza. 29: 1-68.

Singh, B., Mishra, M.K. and Naik, R.K. (2010). Genetic diversity among some traditional aromatic rice (Oryza sativa $L$ ) varieties of orissa. Indian Journal of Agricultural Research. 44(2): 141-145.

Tirkey, A., Sarawgi, A.K. and Subbarao, L.V. (2013). Studies on genetic diversity invarious qualitative and quantitative characters in rice germplasm. Indian Journal of Plant Genetic Resources. 26(2): 132-137.

Umarani, E., Radhika, K., Padma, V. and Rao, S.L.V. (2017). Agromorphological characterization of rice (Oryza sativa L.) landraces based on DUS descriptors. Int J Pure App Biosci. 5: 466-475. 